\title{
Direct Mapping from Body Surface Potentials to Cardiac Activation Maps Using Neural Networks
}

\author{
Amel Karoui ${ }^{1,2,3}$, Mostafa Bendahmane ${ }^{2}$, Nejib Zemzemi ${ }^{1,3}$ \\ ${ }^{1}$ INRIA Bordeaux sud-ouest, France \\ ${ }^{2}$ Institute of Mathematics of Bordeaux, France \\ ${ }^{3}$ IHU-Liryc Bordeaux, France
}

\begin{abstract}
Cardiac mapping is an important diagnostic tool to understand cardiac arrhythmias caused by an electrical disorder. One of its procedures is the activation mapping which consists on generating a map of the depolarization sequence. This map is constructed from the electrograms (EGMs). These latter signals are either recorded invasively through catheterization or noninvasively reconstructed using inverse methods. Our purpose is to evaluate the performance of artificial neural networks on reconstructing activation maps noninvasively directly from body surface potentials (BSPs) without providing the electrograms. We suggest a basic architecture of a neural network. The method is evaluated using simulated atrial data.
\end{abstract}

\section{Introduction}

Activation mapping has been a standard tool of cardiac arrhythmia clinical diagnostic. In clinical routine, it consists on approximating the activation times (ATs) from invasively measured electrograms (EGMs) either from a unipolar signal by determining the point of maximum negative slope (IDT : intrinsic deflection time) or from a bipolar signal, at the point of maximal signal amplitude [1]. This technique is known to have some drawbacks: it is essentially invasive.

To avoid this issue, Electrocardiographic imaging (ECGi) suggests to reconstruct the electrical activity of the heart from non-invasively recorded body surface potentials (BSPs) and a heart-torso geometry reconstruction based on CT-Scan images using computational methods. However, this inverse problem is known to be ill-posed and requires applying regularization techniques on the solution. This yields smoothed EGMs which makes it difficult to determine ATs, peak points of the electrical signals.

Some research studies have suggested lately solving the inverse problem directly in terms of activation times without using the EGMs [2-6]. In this paper, we propose to do so by using Artificial neural networks which is, to our knowledge, has not been suggested yet.

\section{Methods}

\subsection{Neural Network architecture}

We propose here a classical architecture of a neural network constituted of linear layers and the non linear activation function ReLU (Rectified Linear Unit) as a tool of cardiac activation mapping without using the EGMs which is commonly represented in the state-of-the-art. The network architecture is represented in Figure 1. To train our network, we used the mean squared error as an optimization criterion and the stochastic gradient descent as an optimization algorithm. K-fold cross validation [7] is used to evaluate the model performance on unseen data. It generally results on a less biased estimation of the target. Here, the parameter $\mathrm{K}$ is fixed equal to 4 . The procedure consists on splitting the dataset on a training-validation dataset and a testing dataset. Then, $\mathrm{K}$-fold cross validation is applied on the training-validation dataset. In fact, this latter is splitted into $\mathrm{K}$ groups. Each unique group is once kept as a validation dataset and all the remaining ones are used for training the model. In the end, the trained models are evaluated over the testing dataset. The training phase ends when the mean squared error computed over the validation dataset stops improving.

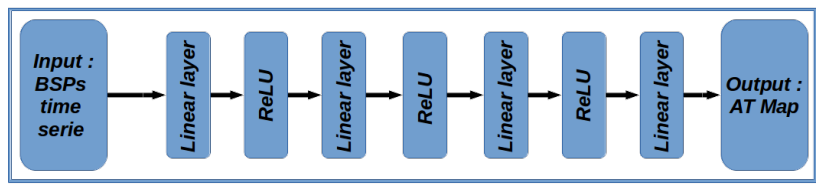

Figure 1: Representation of the neural network architecture 


\subsection{Dataset}

We build a synthetic database of 101 simulations of BSPs and their correspondant AT maps. Each sample of BSPs and AT map corresponds to a stimulation location represented in Figure 2 by red gaussian spheres. We use the monodomain reaction-diffusion model to simulate the electrical wave propagation inside the heart. In order to simulate the BSPs, we first need to compute the extracellular potential in the heart (EGMs). Then, we use a Laplace equation on the torso with a Dirichlet boundary condition on the heart-torso interface to compute the BSPs. For more details, see [8]. Activation times are derived from the simulated EGMs by determining the IDT (Intrinsic Deflection Time) at each point of the atria mesh. Let $u_{i}(t)$ be the unipolar signal at point $X_{i}$ at time $t$, the IDT $\hat{T}_{i}$ is :

$$
\hat{T}_{i}=\arg \min _{t \in[0, T]} \frac{d u_{i}(t)}{d t},
$$

where $T$ is the simulation duration. Here, we only consider the electrical activation in the atria. The finite element discretization of the realistic 3D atria-Torso geometry contains 264 nodes for the torso and 1994 nodes in the atria surface. Each sample contains 400 time steps.

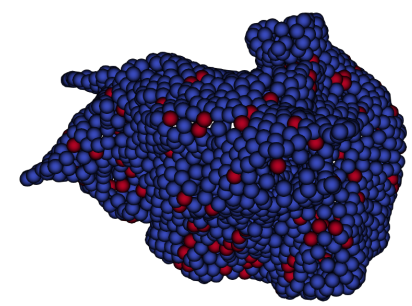

Figure 2: Atria mesh : red dots are the stimulation locations

\subsection{Evaluation metrics}

To assess the precision of reconstructed activation maps, a point-based absolute activation time error (AATE) was computed as the absolute value of the difference between the activation times at each point of the atria mesh. Given $A T_{i, j}^{e}$ the exact activation time at point $j$ of the simulation $i, A A T E_{j}^{i}$ can be expressed as follows :

$$
A A T E_{j}^{i}=\left|A T_{i, j}^{e}-A T_{i, j}^{c}\right|
$$

where $A T_{i, j}^{c}$ is the computed activation time at point $j$ of the simulation $i$. Then, an average all over the mesh is computed and represented in the results section. Linear correlation coefficients are also computed between each activation time map pair for every simulation. Finally, a linear regression is built between reconstructed and reference activation times for each simulation combined with a statistical analysis based on p-value and standard error(stde).

\section{Results}

In this section, results are represented only for the testing dataset.

\subsection{Activation time maps reconstruction}

Reconstructed activation maps using the neural network result in a mean absolute activation time error of $4.69 \mathrm{~ms}$ for the different 12 test simulations. Mean correlation coefficient computed over the same testing dataset reaches $97.8 \%$. Examples of reconstructed activation maps are represented in Figure 3 with the corresponding reference activation maps. In this figure, the mean absolute activation time errors computed over all the mesh points are $4.15 \mathrm{~ms}, 3.68 \mathrm{~ms}, 3.34 \mathrm{~ms}$ and correlation coefficients between AT maps are $0.98,0.98,0.99$, respectively from top to bottom. Figure 4 shows a linear regression of

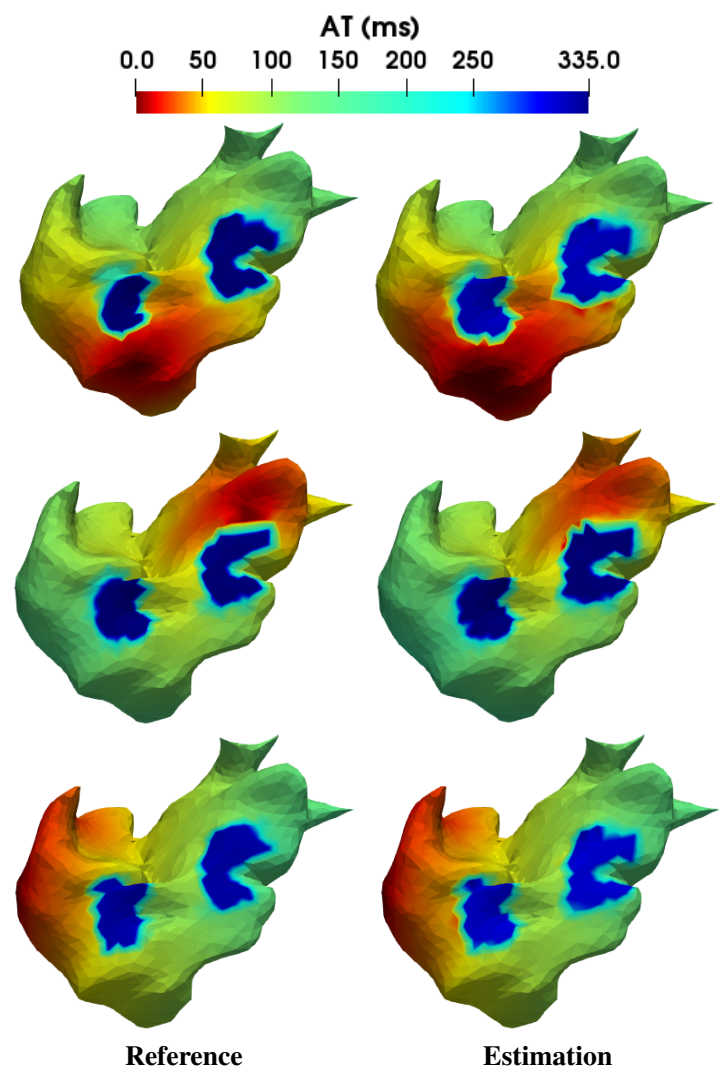

Figure 3: Reference and estimated AT maps of the testing dataset using the neural network.

the estimated AT map with respect to the reference data 
of some testing simulations. In average, it results in a slope of 0.985 and a standard error equal to 0.004 which corresponds to a good fit between the estimated and the reference data.
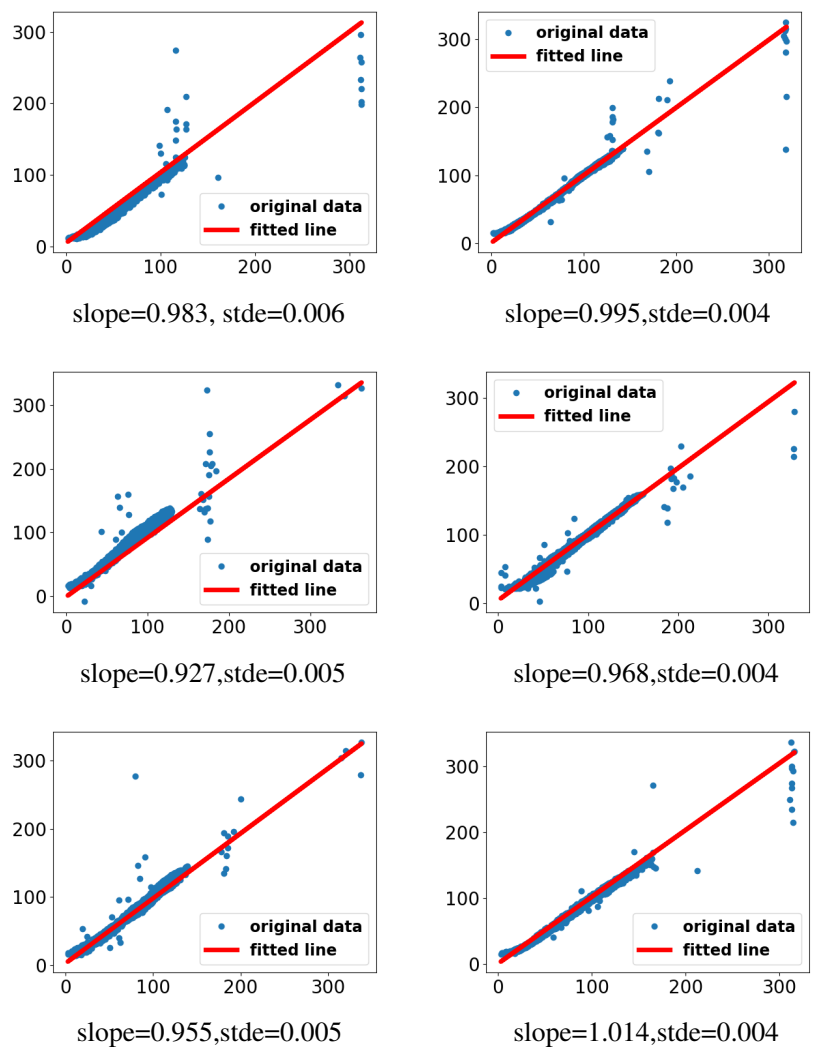

Figure 4: Linear regression between estimated and reference ATs for 6 different simulations of the testing dataset.

\subsection{Robustness analysis}

Data errors often occur in electrocardiographic imaging (ECGi) and BSP recording processes. This is generally due to physiological model errors and/or measurement procedure errors. Thus, a noise effect evaluation is presented in this study. It consists on assessing the model using the testing BSP signals combined with an additive Gaussian noise generated with different noise level values going from $5 \%$ to $50 \%$.

Table 1 shows the evolution of the mean absolute activation time error and correlation coefficient over the testing dataset with respect to the noise level added to the input data. We observe that AATE and CC vary slightly which confirms that our model is strongly robust against additive Gaussian noise.
Table 1: Mean AATE and CC with respect to noise level value

\begin{tabular}{lrrrrr}
\hline \hline$L_{\text {noise }}(\%)$ & 5 & 10 & 20 & 30 & 50 \\
\hline Mean AATE (ms) & 4.65 & 4.60 & 4.67 & 4.69 & 4.70 \\
Mean CC (\%) & 97.84 & 97.84 & 97.83 & 97.84 & 97.81 \\
\hline \hline
\end{tabular}

\section{Discussion and conclusion}

In this paper, we suggest an artificial neural network based approach for cardiac activation mapping. The primitive idea was to build a method for non-invasively reconstructing activation time maps directly from a BSP time serie. This problem is nonlinear which makes it difficult to modelize. So, neural networks can be a good candidate to solve such a problem which has been confirmed by this study. The estimation results are very promising since we reach an optimal value of correlation coefficient $99 \%$ and a weak p-value ( $p \leq 0.0001)$. The model is built and evaluated using almost perfect simulated data with a single pacing site for each simulation. This can be an initiation to other studies where data is more complex and realistic. Moreover, since cardiac activation mapping is a diagnostic tool of cardiac diseases, our model would be more credible if trained and tested using data illustrating some specific cardiac pathologies. A robustness analysis against noise was also conducted. It shows that the model is robust against eventual additive Gaussian noise. Other robustness tests would be the subject of future works such as robustness against electrode misplacement and geometrical modeling errors. Future studies aim to validate our model using clinical data and compare its performance to the state-of-the-art methods. Nevertheless, the challenge of standardizing the heart-torso geometry between patients remains current. Besides, the training database should be sufficiently rich to allow making accurate deductions.

\section{References}

[1] Zipes D, Jalife J. Cardiac electrophysiology: From cell to bedside, 2009 ed, 2009.

[2] Wang L, Zhang H, Wong KC, Liu H, Shi P. Physiologicalmodel-constrained noninvasive reconstruction of volumetric myocardial transmembrane potentials. IEEE Transactions on Biomedical Engineering 2009;57(2):296-315.

[3] Han C, Liu Z, Zhang X, Pogwizd S, He B. Noninvasive threedimensional cardiac activation imaging from body surface potential maps: a computational and experimental study on a rabbit model. IEEE transactions on medical imaging 2008; 27(11):1622-1630.

[4] He B, Li G, Zhang X. Noninvasive three-dimensional activation time imaging of ventricular excitation by means 
of a heart-excitation model. Physics in Medicine Biology 2002;47(22):4063.

[5] Van Dam PM, Oostendorp TF, Linnenbank AC, Van Oosterom A. Non-invasive imaging of cardiac activation and recovery. Annals of biomedical engineering 2009;37(9):1739-1756.

[6] Han C, Pogwizd SM, Yu L, Zhou Z, Killingsworth CR, He B. Imaging cardiac activation sequence during ventricular tachycardia in a canine model of nonischemic heart failure. American Journal of Physiology Heart and Circulatory Physiology 2014;308(2):H108-H114.

[7] Refaeilzadeh P, Tang L, Liu H. Cross-Validation. Boston, MA: Springer US, 2009; 532-538.

[8] Zemzemi N, Dubois R, Coudière Y, Bernus O, Haïssaguerre M. A machine learning technique regularization of the inverse problem in cardiac electrophysiology. In CinCComputing in Cardiology Conference. IEEE, 2013; 285312.

Address for correspondence:

Amel Karoui

INRIA, 200 Avenue de la Vieille Tour, 33405 Talence, France

amel.karoui@inria.fr 